Pedagogías de
la lengua 


\title{
Enunciación
}

\section{Artículo de INVESTIGACIÓN \\ Las tutorías de escritura académica: estrategias didácticas de una tutora novel}

\author{
Academic writing tutorials: teaching strategies of a novice tutor \\ Juan Antonio Núñez Cortés*
}

\section{Resumen}

Las tutorías de escritura son una iniciativa de alfabetización académica en muchas universidades del mundo y en los últimos años han proliferado en universidades del ámbito hispano, en general a través de los centros de escritura u otros programas o proyectos de enseñanza de la escritura académica. El presente trabajo tiene como objetivo dar cuenta del tipo de estrategias didácticas que ponen en práctica los tutores noveles durante las tutorías. Para ello, mediante una investigación de corte cualitativo, se ha realizado un análisis de contenido de las transcripciones de cuatro tutorías grabadas en audio. Los textos analizados se obtuvieron a partir de cuatro tutorías ofrecidas por una tutora de $4^{\circ}$ año de carrera a estudiantes recién ingresados en la universidad. Las estrategias se han clasificado en tres tipos: instructivas, cognitivas y motivacionales. Los resultados muestran un predominio de estrategias cognitivas, si bien la secuencia de estrategias más utilizada (lectura en voz alta del tutor - pregunta - solución del error - explicación - elogio) da cuenta de una tendencia hacia un modelo de tutoría centrada en el tutor y el producto del texto. Esto muestra la importancia de una formación modelada de los tutores a partir de la reflexión sobre lo realizado en tutorías ajenas y propias, al margen de la instrucción explícita sobre la didáctica del proceso de escritura y de los géneros discursivos académicos. Debatir sobre estas estrategias, asimismo, puede ser útil en actividades de revisión entre pares y en tutorías académicas entre profesores universitarios y estudiantes.

Palabras clave: escritura, tutoría, universidad.

\begin{abstract}
Writing tutorials are an academic literacy initiative in many universities around the world and in recent years they have proliferated in universities in the Hispanic field in general through writing centers or other programs or projects for teaching academic writing. The present work aims to account for the type of didactic strategies that the new tutors put into practice during the tutorials. To this end, through qualitative research, a content analysis of the transcripts of four audio-recorded tutorials has been carried out. The analyzed texts were obtained from four tutorials offered by a 4th career tutor to students recently entered the university. The strategies have been classified into three types: instructional, cognitive and motivational. The results show a predominance of cognitive strategies, although the most used sequence of strategies (reading aloud from the tutor - question - solution of the error - explanation - praise) shows a trend towards a tutoring model focused on the tutor and the text product. This shows the importance of a modeled training of the tutors from the reflection on what has been done in external and own tutorials, apart from the explicit instruction on the didactics of the writing process and the academic discursive genres. Discussing these strategies can also be useful in peer review activities for the same course and in academic tutoring between university professors and students.
\end{abstract}

Keywords: writing, tutoring, university.

* Profesor del Departamento de Filologías y su Didáctica de la Facultad de Formación de Profesorado y Educación de la Universidad Autónoma de Madrid. Doctor en Educación por la Universidad Autónoma de Madrid. Coordinador del Centro de Escritura. Correo electrónico: juanantonio.nunnez@uam.es

Cómo citar: Núñez Cortés, J. A. (2020). Las tutorías de escritura académica: estrategias didácticas de una tutora novel. Enunciación, 25(2), 176-190. https://doi. org/10.14483/22486798.16563

Artículo recibido: 26 de junio de 2020; aprobado: 5 de agosto de 2020 


\section{Introducción}

Ante las dificultades y desafíos de escritura a los que se enfrentan los estudiantes universitarios, son diferentes las iniciativas que se pueden Ilevar a cabo para solventarlos. Así, se ofrecen asignaturas de expresión escrita al comienzo de la carrera, talleres sobre géneros discursivos académicos o repositorios con materiales que facilitan y orientan sobre la norma lingüística, las fases del proceso de escritura, los géneros académicos o sobre convenciones disciplinares como los sistemas de citación, entre otras. También, son conocidas las asesorías y el trabajo conjunto entre profesores especialistas en didáctica de la escritura y profesores de las asignaturas, así como propuestas de escritura a través del currículum o en las disciplinas. Además, otra de las iniciativas para atender a los estudiantes son las tutorías de escritura ofrecidas en el marco de un centro o programa de escritura y también en proyectos de innovación docente (Núñez y Errázuriz, 2019). Estas tutorías parten de la premisa de que una atención individualizada o en pequeños grupos de estudiantes ha de entenderse como condición en ocasiones imprescindible para alcanzar la calidad y la equidad de la educación basada en la inclusión. Entre los motivos destacan las características personales de cada estudiante y la heterogeneidad en sus demandas e intereses; así como la falta de formación en enseñanza de la escritura en las disciplinas de muchos profesores.

Así pues, cabe aclarar al comienzo de este trabajo el concepto de tutoría de escritura. Al respecto, hay que diferenciarla de la tutoría tradicional impartida por un profesor universitario a un estudiante en el marco de una iniciativa institucional -que sirve de vínculo con la propia universidad-, o de una iniciativa didáctica, y estar integrada en el proceso de aprendizaje de una asignatura (Castellà y Aliagas, 2016). También es preciso mostrar las divergencias entre una tutoría de escritura y la revisión entre pares. El segundo de los casos responde a un proceso planificado de escritura de una secuencia de aprendizaje en una asignatura y los pares simétricos no han tenido por qué recibir formación sobre el proceso de escritura (Chois et al., 2017). En todo caso, las tutorías de escritura cuyos tutores son estudiantes -puede haber también tutores profesores- son consideradas como tutorías entre iguales. En ellas, quienes cuentan con mayor experticia acompañan durante el proceso de escritura de un texto a estudiantes menos duchos (Alzate y Peña, 2010) y, generalmente, los tutores son de cursos y edades superiores. Además, han recibido una formación explícita para desempeñarse como tutores de escritura, sea en el marco de un centro de escritura o en el de otras iniciativas de programas o proyectos de innovación centrados en la enseñanza de la escritura. Así pues, la relación que se establece entre el tutor y el tutorado es asimétrica, si bien esto no impide un aprendizaje mutuo en tanto no hay calificación del texto trabajado y los participantes son estudiantes universitarios que están viviendo experiencias parecidas (Chois et al., 2017).

Dicho esto, se pueden definir las tutorías de escritura como una iniciativa pedagógica en la que un tutor (estudiante o profesor) formado acompaña a un(os) estudiante(s) durante el proceso de escritura de un texto académico o profesional, con el objetivo de reflexionar sobre su escritura y así participar en su mejora como escritor(es). Por esto, es necesario incidir en la idea de North (1982) de que el tutor no es un editor ni corrector de textos. Por el contrario, entre sus funciones está hacer que el tutorado se sienta dueño de su trabajo y que formule sus propias preguntas y respuestas sobre el texto (Alzate y Peña, 2010), ayudar a que aprenda sobre la escritura y colaborar en el cambio del conocimiento previo del estudiante en función de las demandas de la tutoría (Boyd, 2013). Asimismo, es importante señalar que el tutor no debe imponer su estilo de escritura, ni considerar que el suyo es el único adecuado. Tiene, por tanto, que aceptar el estilo ajeno y colaborar en la construcción o definición de la identidad del estudiante como 
escritor. Por todo ello, en el espacio de la tutoría desempeñará diferentes papeles como el de lector, comentarista, colaborador, aprendiz, consejero, etc. (Ryan y Zimmerelli, 2006).

En cuanto a los fundamentos pedagógicos de las tutorías de escritura, estas se inspiran en las teorías constructivista y sociocultural de Piaget (1970) y Vygotsky (1978), respectivamente. En este sentido, han de entenderse las tutorías como las concibe Monty (2013): un espacio en donde se propicia la zona de desarrollo próximo (ZDP), en donde gracias a la interacción verbal entre estudiante y tutor, el primero lleva a cabo una tarea que no habría podido resolver por sí solo (Castellà y Aliagas, 2016). Esto, a su vez, conlleva una transformación del conocimiento escribiendo y no una mera reproducción memorística de los saberes (Scardamalia y Bereiter, 1992). Al respecto, Castellà y Aliagas llaman la atención sobre las experiencias exitosas de tutorías de escritura tanto en España como en Latinoamérica.

Estas experiencias dan cuenta de los aspectos positivos o beneficios que se derivan de las tutorías. Algunos que muestran las investigaciones (Alzate y Peña, 2010; Bach y Montané, 2016; Castellà y Aliagas, 2016; Chois et al., 2017; Durán, 2006; Kirchhoff, 2016; Roland, 2008) son los siguientes: a) favorecen la integración en la comunidad académica de estudiantes de los primeros cursos, de aquellos con dificultades de escritura y de quienes tienen diferentes necesidades; b) ofrecen la posibilidad de hablar sobre géneros discursivos académicos, lo que influye en el desarrollo de la alfabetización académica de los estudiantes; c) incrementan la motivación hacia la mejora y gusto por la escritura y, en consecuencia, el rendimiento académico; d) constituyen una enriquecedora experiencia de aprendizaje tanto para los estudiantes como para los tutores, pues los segundos toman conciencia de su propio aprendizaje al activar procesos de metacognición; e) desarrollan valores de solidaridad y sociabilidad así como contribuyen al enriquecimiento personal, académico y profesional de los tutores.
Por otro lado, se ha afirmado que no hay dos tutores iguales, que cada cual tiene una visión única de la tutoría (Dinitz y Kiedaisch, 2003) y que la adscripción del tutor a la misma disciplina del estudiante es determinante (Cromley y Azevedo, 2005). Asimismo, se han identificado diferentes tipos de tutorías (García-Arroyo y Quintana, 2016; Roldán y Arenas, 2016; Wingate, 2005). En líneas generales, se pueden destacar dos clases opuestas, no exentas de matices en función de la situación. Las primeras se centran en el estudiante y en el proceso, y así es el tutorado quien lee el texto, plantea dudas de escritura y recibe las recomendaciones del tutor. Por el contrario, en las tutorías centradas en el tutor -y el producto- es este quien lee el texto, señala los errores y formula preguntas que ahora el estudiante responde. No obstante, como se ha indicado, estos son dos modelos dicotómicos y entre ellos pueden darse múltiples circunstancias. Quizá valdría abogar por un modelo de tutoría de escritura colaborativa, en la que el tutor participa en la resolución de los problemas de escritura y acompaña al estudiante en la toma de sus propias decisiones. Al respecto, los tutores durante las tutorías tienden a dar importancia a fomentar una buena relación con los estudiantes y la de estos con sus propios textos, el trabajo conjunto entre los dos participantes y transmitir sus reflexiones a los estudiantes (Harrington, $\mathrm{O}^{\prime} \mathrm{Neill}$ y Bakhishi, 2007).

En cuanto a la retroalimentación sobre la escritura, las investigaciones se han centrado sobre todo en el tipo de comentarios que hacen los profesores en sus devoluciones escritas (Natale, 2014, 2016) o los propios estudiantes en actividades de revisión por pares insertas en las asignaturas. Algunos trabajos muestran cómo la retroalimentación centrada en sugerencias y preguntas genera un cambio más significativo que el producido por comentarios directivos (Álvarez y Difabio de Anglat, 2018; Álvarez, Espasa y Guasch, 2011). No obstante, en general, los estudiantes suelen considerar que los comentarios de sus profesores podrían ser más útiles, pues muchos son demasiado generales 
y se centran en lo negativo (Weaver, 2006). Por otro lado, Corcelles, Cano, Bañales y Vega (2013) señalan que los estudiantes pares retroalimentan más que sus profesores, si bien predominan comentarios sobre aspectos formales frente a aquellos vinculados con la coherencia y cohesión del texto, más propios de los profesores.

Tal y como se ha mencionado anteriormente, la formación de los tutores es un requisito para que puedan ofrecer tutorías. Esta formación se basa, normalmente, en varios módulos que buscan: a) presentar la estructura, las iniciativas y los recursos de que dispone el proyecto, programa o centro de escritura en donde se realizan las tutorías; b) reflexionar sobre el proceso de escritura y los géneros discursivos académicos; c) mostrar las bases teóricas que sustentan el modelo de enseñanza de la escritura adoptado o adaptado por el proyecto, programa o centro de escritura; d) ofrecer orientaciones y estrategias para el desarrollo de la tutoría y sobre cómo actuar en función del estudiante y sus demandas; e) analizar intervenciones de tutores expertos y grabaciones de los propios tutores en formación. Al respecto de este último módulo, en diferentes contextos se ha mostrado que es una práctica beneficiosa (Gilewicz y Thonus, 2003; Errázuriz, 2017). Esto coincide con la idea de ofrecer a los tutores una formación individualizada (Pigliacelli, 2017). Por otro lado, abundan en el ámbito anglosajón los manuales de formación de tutores de escritura (Fitzgerald y lanetta, 2016; Murphy y Sherwood, 2008; Ryan y Zimmerelli, 2006) en los que se presentan recomendaciones o consejos de toda índole y atañen a diferentes aspectos de las tutorías como el espacio o la manera de sentarse, el modo de comenzar o concluirlas, el establecimiento de los objetivos, etc. También hacen referencia a las estrategias didácticas que han de emplear los tutores.

Estas han sido caracterizadas y sistematizadas en diversos trabajos (Caldwell, Stapleford y Tinker, 2018; Cromley y Azevedo, 2005; Eastmond, 2019; Mackiewicz, 2004, 2005; Mackiewicz y Thompson, 2014, 2018; Roldán y Arenas, 2016;
Thompson, 2009). De entre todas las propuestas, es especialmente interesante la de Mackiewicz y Thompson $(2014$; 2018) por su especificidad en su categorización. Las investigadoras establecen dos niveles de análisis. En el primero, el macronivel, identifican tres fases: apertura, desarrollo y cierre; en el segundo, el micronivel, atienden a tres categorías: instrucción, andamiaje cognitivo y andamiaje motivacional.

La instrucción es en esencia directiva, pues se dice, se sugiere o se explica qué hacer, y no se genera un espacio para que los estudiantes encuentren soluciones por sí mismos. Por el contrario, las estrategias de andamiaje cognitivo favorecen la búsqueda y encuentro de soluciones a través de la incitación al pensamiento y el diálogo entre el tutor y el estudiante. La propuesta de Mackiewicz y Thompson, presentada en español por Molina-Natera (2019), incluye ocho estrategias: jalonar, leer en voz alta, responder como lector u oyente, referirse a un tópico previo, forzar una elección, incitar, insinuar o sugerir, y demostrar. Cabe señalar que las estrategias de andamiaje cognitivo se asocian con los tutores más experimentados (Cromley y Azevedo, 2005). Por último, las estrategias de andamiaje motivacional se centran en el afecto hacia el estudiante para así fomentar su participación y confianza, y ayudar al tutor a construir una buena relación con el tutorado. Proponen cinco estrategias motivacionales: mostrar interés, elogiar, reforzar la posesión y control del tutorado, ser optimista o mostrar humor, y mostrar simpatía y empatía.

Dicho esto, cabe indagar sobre las estrategias didácticas que se utilizan en las tutorías de escritura y, en consecuencia, también qué estrategias conforman una buena tutoría o tutoría exitosa. Parece que esta variable habrá de encontrar correlación con aspectos como a) la formación recibida por el tutor, su nivel de experticia y sus estudios; b) el nivel de estudios del estudiante, pues no es lo mismo uno recién matriculado en la universidad que otro, por ejemplo, de maestría; c) el objetivo de la tutoría, el género discursivo trabajado y la fase del proceso de escritura del texto; d) la 
percepción y el grado de satisfacción de la tutoría sobre todo por parte del estudiante, pero también del tutor; y, por último, e) la mejora de la calidad del texto -pese a la idea clara de que el objetivo es siempre hacer mejores escritores y no mejores escritos- o, cuando menos, lo aprendido por el estudiante. En esta línea, Thonus (2002), por ejemplo, relacionó algunas características de las tutorías con entrevistas a estudiantes nativos y no nativos de inglés, y concluyó que los comentarios no directivos, al igual que en la retroalimentación escrita de los profesores y la ayuda del tutor caracterizaban las tutorías exitosas.

Así pues, el objetivo principal de esta investigación es conocer las estrategias didácticas que utilizan los tutores en las tutorías de escritura tras un modelo específico de formación. En concreto, se pretende conocer el tipo de estrategias (instructivas, cognitivas y motivacionales) así como su cantidad en un tutor novel durante sus primeras tutorías.

\section{Metodología}

\section{Método}

Para llevar a cabo esta investigación se ha hecho uso del análisis de contenido, una metodología de corte cualitativo. Este enfoque ha sido utilizado previamente por otras investigaciones que han analizado las tutorías de escritura en centros de escritura (Cromley y Azevedo, 2005; Eastmond, 2019; Mackiewicz, 2004, 2005; Mackiewicz y Thompson, 2014, 2018; Roldán y Arenas, 2016; Thompson, 2009) por considerarse adecuado para comprender la dinámica propia de las tutorías de escritura entre tutor y estudiante.

\section{Muestra}

En la investigación se han analizado las transcripciones de cuatro tutorías individuales de media hora grabadas en audio y realizadas por una sola tutora y cuatro estudiantes. Tanto los estudiantes como la tutora fueron informados al comienzo de las tutorías de que estas serían grabadas para fines de investigación y que durante el análisis y difusión de los resultados se guardaría el anonimato. Los participantes dieron consentimiento para realizar la grabación. Los estudiantes que participaron en las tutorías cursaban primero del Grado en Maestro(a) de Educación Primaria de la Facultad de Formación de Profesorado y Educación, de la Universidad Autónoma de Madrid. Asimismo, los cuatro estudiantes llevaron a las tutorías textos argumentativos que se pueden adscribir al género discursivo miniensayo (Uribe, Mateo, Agosto y Álvarez, 2020). A los estudiantes de dos grupos de la asignatura Habilidades de Comunicación Oral y Escrita, que se cursa en el primer semestre de esta titulación, se les planteó como tarea la escritura de un miniensayo y, con el fin de familiarizarse al comienzo de la carrera con el centro de escritura de la facultad y sus iniciativas, se les ofreció la posibilidad de solicitar una tutoría para revisar el borrador del texto. Por tanto, en las cuatro tutorías el perfil del estudiante, el tipo de texto y la fase del proceso de escritura es similar.

La tutora cursaba cuarto año, el último, del Grado en Maestro(a) de Educación Infantil en la misma facultad, por lo que estaba familiarizada con las convenciones disciplinares y las formas de decir propias del ámbito educativo. Además, la tutora en el primer semestre de primero de carrera también había cursado la misma asignatura que los estudiantes que solicitaron la tutoría. En la actualidad, los tutores de la facultad realizan su tarea voluntariamente. Como criterios de selección se tienen en consideración una carta motivacional, sus destrezas en expresión escritura y una entrevista personal con el responsable del centro de escritura. La formación recibida por la tutora en el centro de escritura contempló tres módulos:

1. El centro de escritura. En este módulo se presenta el centro de escritura, su estructura, sus funciones, sus iniciativas y los recursos de los que dispone. La formación se 
lleva a cabo en una sesión grupal de dos horas con los tutores noveles al comienzo del curso académico, y se hace hincapié en la importancia de familiarizarse con los recursos que ofrece el centro a través de su página web y de la biblioteca de la universidad.

2. El proceso de escritura y los géneros discursivos académicos. Este módulo consta, a su vez, de tres partes. La primera es un seminario sobre la enseñanza de la escritura de diez horas de duración y que se centra en la didáctica de las fases del proceso de escritura y en mostrar experiencias de otros centros de escritura. La segunda es un taller de escritura de géneros discursivos académicos de diez horas de duración en donde se exponen su estructura y características prototípicas, así como errores frecuentes de expresión escrita de estudiantes universitarios y cómo abordarlos.

3. Las tutorías. El último módulo se divide en cuatro apartados. En primer lugar, en una reunión de dos horas se presentan y comentan los documentos Manual del tutor de escritura y Orientaciones para una buena tutoría. En segundo lugar, la tutora en formación observa dos tutorías de media hora de duración de un tutor experimentado y, posteriormente, se comentan y se reflexiona en torno a lo sucedido en las tutorías. La tercera iniciativa consiste en una tutoría en la que el tutor experto tutoriza a la tutora novel a partir de un texto escrito por ella. Por último, en cuarto lugar, la tutora novel realiza dos tutorías con estudiantes de media hora de duración y es observada por el tutor formador. Al igual que en la observación del tutor experto, la tutora novel y el formador comentan y evalúan la tutoría. Hay que señalar que en este caso se hacen comentarios generales sobre las tutorías, pero no se hace un análisis pormenorizado de las estrategias didácticas que pone en práctica la tutora a través de video o audio y sus transcripciones. Esto es lo que se hace en el presente trabajo.

\section{Instrumento de análisis}

A partir de una lógica de análisis inductiva-deductiva, como resultado, por un lado, del proceso hermenéutico de la lectura analítica de los textos transcritos a partir de las grabaciones en audio y, por otro lado, también a partir de las investigaciones precedentes ya citadas (Caldwell, Stapleford y Tinker, 2018; Cromley y Azevedo, 2005; Eastmond, 2019; Mackiewicz, 2005; Mackiewicz y Thompson, 2014, 2018; Roldán y Arenas, 2016; Thompson, 2009), se establecieron una serie de estrategias didácticas (tabla 1). Estas se han agrupado en tres tipos de estrategias (instructivas, cognitivas y motivacionales) y coinciden en general con la propuesta de Mackiewicz y Thompson (2018) al clasificarlas en instrucción, andamiaje cognitivo y andamiaje motivacional. Cabe indicar que, frente a la propuesta de las investigadoras, algunas de sus estrategias se han matizado y otras son nuevas; asimismo, se ofrecen ejemplos propios. En este sentido, hay que señalar que una de las estrategias que forman parte de las estrategias cognitivas, la solución, se puede considerar de carácter instructivo. No obstante, pese a que en el andamiaje cognitivo se busca que el estudiante encuentre una solución, se ha visto oportuno incluirlo en esta categoría pues en estos casos el tutor no hacía explícita a través de una sugerencia u orientación la indicación, sino que señalaba directamente la respuesta ante el error, duda o problema

Finalmente, estas categorías con sus correspondientes estrategias se han sometido a un proceso de codificación y sistematización. Se codificaron en el software de análisis cualitativo MAXQDA Analytics Pro 2020, para asignar cada una de las estrategias a sus categorías y así conocer la frecuencia y relación entre cada una de ellas. 
Tabla 1. Estrategias didácticas de las tutorías de escritura

\section{Estrategias instructivas}

\begin{tabular}{ll}
\hline Explicación & $\begin{array}{l}\text { Se ofrecen razones, ilustraciones o ejemplos de los consejos ofrecidos. Todo lo que has quitado lo puedes } \\
\text { resumir, o sea, puedes decir la misma idea con menos palabras. }\end{array}$ \\
\hline Orientación & Se indica o aconseja al estudiante sobre lo que ha de hacer de forma directa, no mitigada. No repitas esta idea. \\
\hline Sugerencia & $\begin{array}{l}\text { Se indica o aconseja al estudiante sobre lo que ha de hacer de forma mitigada, minimizando así la indicación } \\
\text { (p. ej., a través de la cortesía negativa). Yo la quitaría. }\end{array}$ \\
\hline
\end{tabular}

\section{Estrategias cognitivas}

$\begin{array}{ll}\text { Completamiento } & \text { Se incita a responder al estudiante proporcionándole una respuesta o enunciado parcial y así reducir las posi- } \\ \text { bles respuestas (sc. rellenar un espacio en blanco). Tutor: "Trasmitirle una idea .... planteada....". Estudiante: "E }\end{array}$ una reunión".

Comprobación $\quad$ Se comprueba la comprensión del estudiante de algo ya tratado mediante una interrogación o la solicitud de una explicación. ¿Entiendes? ¿Me explico?

Elección Se ofrecen varias alternativas para que se escoja una. Estudiante: "No tiene uso, más bien". Tutor: "Está en desuso", "no se utiliza".

Finalidad Se establece o se busca establecer el objetivo de la tutoría. Se recuerda el objetivo de la tutoría. Vale, entonces no te interesa quizá tanto la redacción del texto en sí, como saber reducir la extensión.

Interpretación Se interpreta el texto del estudiante, es decir, se muestra qué se entiende del fragmento referido. Se parafrasea

el texto. Yo, como lectora, al leer esto me planteo si existe otro gimnasio que no sea el de la planta de abajo.

Lectura en voz alta El estudiante lee en voz alta fragmentos de su texto con el objetivo de identificar errores de contenido y/o

del estudiante forma. Tutor: "Pues si te parece lo vas leyendo tú, que así también te puedes ir dando cuenta mejor de los errores". (Estudiante lee su texto).

Lectura en voz alta Se leen en voz alta fragmentos del texto del estudiante para escuchar lo escrito o la consigna de la tarea de

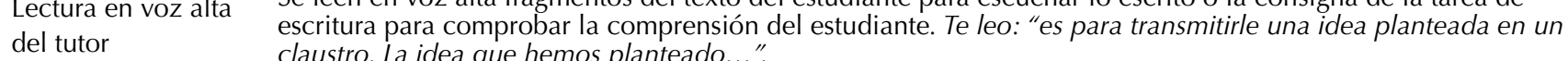
claustro. La idea que hemos planteado...".

\begin{tabular}{ll}
\hline Marcación & $\begin{array}{l}\text { Se señala explícitamente un problema, error o aspecto del texto que ha de atenderse. En esa primera oración } \\
\text { que has escrito hay algo que falta relacionado con la puntuación. }\end{array}$ \\
\hline Soporte & $\begin{array}{l}\text { Se muestra al estudiante un texto modelo, una guía de escritura o una rúbrica para que aprenda a hacer algo. } \\
\text { Mira cómo se plantea en este ejemplo, que es prácticamente igual, pues es un texto argumentativo. }\end{array}$ \\
\hline Pregunta & $\begin{array}{l}\text { Se formula una o varias preguntas que requieren de la respuesta del estudiante. Las preguntas pueden ser espe- } \\
\text { cíficas o generales. ¿Por qué cambias de párrafo? }\end{array}$ \\
\hline Recomendación & $\begin{array}{l}\text { Se recomienda al estudiante el uso de un recurso para que resuelva el problema, error o aspecto tratado. En la } \\
\text { Web del centro de escritura hay un recurso sobre cómo escribir un estado de la cuestión. }\end{array}$ \\
\hline Reescritura & $\begin{array}{l}\text { Se plantea al estudiante que reescriba un fragmento que contiene un problema, error o aspecto que debe ser } \\
\text { atendido. Pues entonces, si no te convence, reescríbelo como me acabas de decir y luego vemos cómo queda. }\end{array}$ \\
\hline Remisión & $\begin{array}{l}\text { Se remite a un tema, error, problema o aspecto del texto ya tratado. Pero eso ya lo hemos mencionado anterior- } \\
\text { mente. }\end{array}$ \\
\hline Reticencia & $\begin{array}{l}\text { Se hace referencia o plantea un problema, error o aspecto del texto mediante una estrategia de cortesía indi- } \\
\text { recta no convencional. Aun así, lo que yo te quería decir es que si tú dices: "porque según como argumentan". } \\
\text { Así no suena muy bien. }\end{array}$ \\
\hline Solución & $\begin{array}{l}\text { Se ofrece la solución al problema, error o aspecto del texto. (El tutor lee el texto e indica el cambio): "El motivo } \\
\text { por el que nos ponemos en contacto con usted es para transmitirle una idea (coma), que hemos debatido en el } \\
\text { claustro...". }\end{array}$ \\
\hline
\end{tabular}

\section{Estrategias motivadoras}

Ánimo/humor Se anima al estudiante a perseverar en la tarea y mejorar el texto. Se utiliza el humor mediante bromas relacionadas con el texto o el contexto de la tutoría. Esa tiene tela. (Tras leer una oración ininteligible, tutor y estudiante se ríen antes y después).

\begin{tabular}{ll}
\hline Elogio & $\begin{array}{l}\text { Se señalan los aspectos positivos del texto o de la resolución de los problemas por parte del estudiante. Mucho } \\
\text { mejor así. Perfecto. }\end{array}$ \\
\hline Empatía & Se expresa la compresión de que la tarea es difícil. Este tipo de texto siempre es complicado. \\
\hline Identificación & $\begin{array}{l}\text { Se hace referencia a experiencias propias relacionadas con la escritura de textos similares. Eso es una duda } \\
\text { que yo también suelo tener a veces. / Claro. Yo lo hago igual. }\end{array}$ \\
\hline Interés & Se muestra el interés por el estado emocional del estudiante. ¿Ves que no es tan difícil? ¿Estás mejor? \\
\hline Refuerzo & $\begin{array}{l}\text { Se incide en que es el estudiante quien debe tomar las decisiones. Entonces, ahora ahí, haces las modificacio- } \\
\text { nes que consideres. / Lo dejo a tu elección. }\end{array}$ \\
\hline Significación & $\begin{array}{l}\text { Se mencionan los aspectos aprendidos o trabajados durante o al final de la tutoría, recapitulando lo tratado. Se } \\
\text { hace referencia a la relevancia que puede tener lo aprendido en la escritura de futuros textos. Así no estaba en } \\
\text { tu texto, esto de la coma entre sujeto y verbo no lo ponías así y ahora sí. }\end{array}$ \\
\hline
\end{tabular}

Fuente: elaboración propia a partir de Mackiewicz y Thompson (2018). 


\section{Resultados}

A partir del análisis realizado, y con relación al objetivo principal de conocer cuáles son las estrategias didácticas más utilizadas en las tutorías de una tutora novel, se puede afirmar que predominan las estrategias cognitivas, con un $52 \%$ del total de intervenciones por parte de la tutora, seguidas de las instructivas $(36,5 \%)$ y las motivacionales $(11,55 \%)$. Esto muestra la conciencia por parte de la tutora de la importancia de que en las tutorías prime el andamiaje cognitivo para así favorecer que los estudiantes encuentren sus propias respuestas a los problemas o dudas que surgen a partir de los textos y colaborar en la búsqueda de soluciones.

En cuanto a las estrategias instructivas, hay que señalar el uso similar de la explicación, la orientación y la sugerencia (tabla 2). En estas estrategias se destaca la tendencia a disminuir gradualmente la orientación mediante la cual se aconseja e indica al estudiante de manera directa lo que ha de hacer. Así, frente a las dos primeras tutorías, en las que la tutora emplea la orientación en 19 y 20 ocasiones, respectivamente, y en 15 en la tercera, en la última llega a utilizarla la mitad de las veces (9).

Las estrategias cognitivas son, como se ha indicado, las más utilizadas y, tras el análisis, se pueden clasificar en tres grupos en función de su uso: abundante, moderado y escaso. En el primer grupo se encuentran las de lectura en voz alta por parte de la tutora, la pregunta y la solución. Destaca, en este caso, la tercera tutoría, pues es en la que en más ocasiones la tutora lee en voz alta y ofrece soluciones; estrategias que se consideran propias de un modelo de tutoría centrado en el tutor y en el producto del texto, y que se asocian con la falta de experiencia. Por otro lado, un uso moderado de estrategias cognitivas respecto del total estaría constituido por la comprobación, la elección, la interpretación, la marcación y la reticencia. Se percibe, por ello, la intención de la tutora de generar en la tutoría un espacio de aprendizaje colaborativo, al hacer lo posible para comprobar la comprensión de las explicaciones que ofrece al estudiante, al señalarle el problema o error del texto de manera mitigada y al ofrecerle varias alternativas para que escoja una. Muestra, por tanto, su interés e intento de contribuir al andamiaje cognitivo del estudiante pese a que predominen estrategias que mitigan esta intención. Por último, en cuanto al último grupo, son las estrategias de completamiento, finalidad, lectura en voz alta por parte del estudiante, recomendación, reescritura, remisión y soporte las que en menos ocasiones se emplea. Si bien todas las estrategias colaboran en el éxito de la tutoría, es cierto que la tutora novel encuentra dificultad en poner en práctica algunas más eficaces para su consecución. Así, algunas como la lectura en voz alta por parte del estudiante, el establecimiento de la finalidad de la tutoría o la propuesta de reescritura del texto con el fin de mejorarlo y reflexionar sobre la nueva propuesta apenas se proponen.

Con relación a las estrategias motivacionales, la más utilizada es el elogio, seguida de la identificación; frente a otras como el ánimo, el refuerzo y la significación. Dos estrategias, la empatía y el interés, no aparecen en ninguna de las tutorías. Por ello, si bien se señalan los aspectos positivos del texto o la buena capacidad para resolver los problemas por parte del estudiante, así como se percibe el interés por dar cuenta de que la tutora ha tenido experiencias de escritura similares; se desatiende el estado emocional del estudiante y no se muestra la complejidad de la tarea de escritura.

Dado que la estrategia cognitiva más utilizada en las tutorías es la lectura en voz alta por parte del tutor, interesa saber cómo se relaciona con el resto de estrategias instructivas, cognitivas y motivacionales. Como se aprecia en la figura 1, cuando el tutor lee, suele utilizar de manera equitativa la sugerencia, la orientación y la explicación. Además, la estrategia se encuentra siempre próxima a las otras estrategias cognitivas más frecuentes (la solución y la pregunta) y al elogio (estrategia 
Tabla 2. Frecuencia de estrategias didácticas en las tutorías de escritura

\begin{tabular}{|c|c|c|c|c|c|}
\hline Códigos & TTN1 & TTN2 & TTN3 & TTN4 & SUMA \\
\hline \multicolumn{6}{|l|}{ Estrategias instructivas } \\
\hline Explicación & 14 & 28 & 19 & 14 & 75 \\
\hline Orientación & 19 & 21 & 15 & 9 & 64 \\
\hline Sugerencia & 19 & 19 & 15 & 20 & 73 \\
\hline \multicolumn{6}{|l|}{ Estrategias cognitivas } \\
\hline Completamiento & & & 4 & 1 & 5 \\
\hline Comprobación & 9 & 8 & 1 & 3 & 21 \\
\hline Elección & 4 & 13 & 6 & 7 & 30 \\
\hline Finalidad & 1 & & & & 1 \\
\hline Interpretación & 3 & 9 & 7 & 2 & 21 \\
\hline Lectura estudiante & & 2 & 1 & & 3 \\
\hline Lectura tutor & 8 & 14 & 32 & 12 & 66 \\
\hline Marcación & 11 & 4 & 3 & 5 & 23 \\
\hline Pregunta & 15 & 13 & 15 & 16 & 59 \\
\hline Recomendación & 1 & 1 & 1 & 1 & 4 \\
\hline Reescritura & 2 & & & 1 & 3 \\
\hline Remisión & 1 & & & & 1 \\
\hline Reticencia & 8 & 5 & 5 & 2 & 20 \\
\hline Solución & 7 & 4 & 25 & 9 & 45 \\
\hline \multicolumn{6}{|l|}{ Estrategias motivadoras } \\
\hline Ánimo/humor & 3 & 1 & & & 4 \\
\hline Elogio & 14 & 16 & 8 & 5 & 43 \\
\hline Identificación & 1 & 6 & 2 & 1 & 10 \\
\hline Refuerzo & 2 & 2 & & 2 & 6 \\
\hline Significación & 1 & 1 & & 2 & 4 \\
\hline I SUMA & 143 & 167 & 159 & 112 & 581 \\
\hline
\end{tabular}

Fuente: elaboración propia.

motivacional). Destaca, asimismo, que no aproveche la oportunidad de la lectura para emplear otras estrategias como la interpretación del texto leído o, tras haber señalado el error, no ofrezca la posibilidad de que el estudiante lo solucione mediante la elección o el completamiento.

A partir de estos datos, se puede afirmar que las estrategias más utilizadas por parte de la tutora novel muestran un modelo de tutoría en donde predomina la explicación de las ideas que la tutora considera que deben quedar claras al estudiante y la pregunta. Además, en general, se han ofrecido las soluciones a las dudas, problemas o errores del estudiante a partir de la lectura en voz alta del tutor, sin dejar de llamar la atención sobre los aspectos positivos. Así, una secuencia prototípica de la tutoría podría ser la que aparece en el ejemplo que se detalla en la tabla 3, extraído de la tutoría 3. 


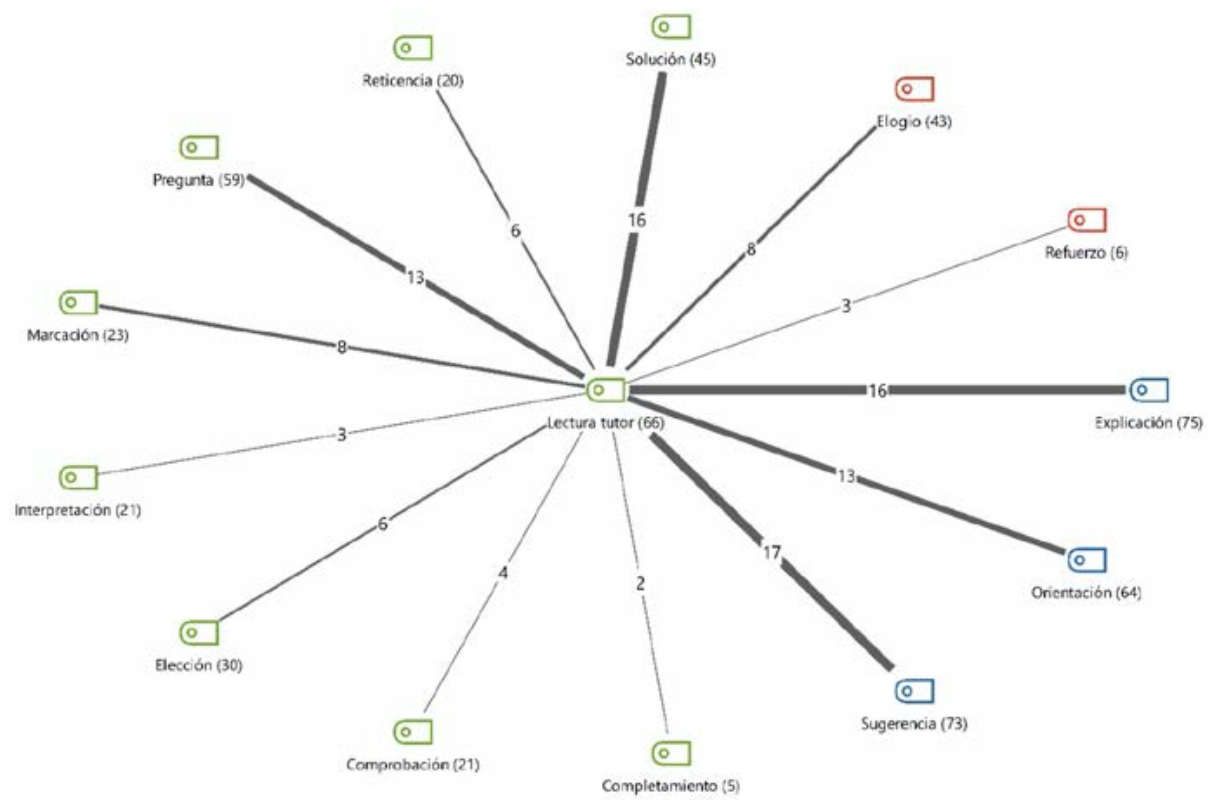

Figura 1. Proximidad de la estrategia de lectura en voz alta del tutor con el resto de estrategias

Fuente: elaboración propia.

Tabla 3. Ejemplo de secuencia prototípica de estrategias didácticas de un tutor novel

\section{Fragmento de tutoría}

T: “Otro motivo es el traslado (coma) ya que entre la ida y la vuelta..."

\section{E: Sí.}

T: ¿A qué te estás refiriendo aquí?

E: Claro, la coma después de traslado.

T: Sí, muy bien.

E: Como con "es decir", ¿no?

T: Eso es. Siempre ten en cuenta que los conectores discursivos van con comas, como en: "En primer lugar, ...".

\section{Estrategia y explicación}

Lectura en voz alta de la tutora y solución.

En esta intervención, la tutora mientras lee el texto del estudiante señala un error que ha detectado.

El estudiante detecta el error gracias a la solución mostrada.

Pregunta

La tutora interroga al estudiante sobre el sentido de la oración a la espera de una respuesta. No hace hincapié en el error solucionado.

El estudiante describe la solución al error, repitiendo la primera indicación de la tutora

\section{Elogio}

La tutora valora la identificación del error por parte del estudiante. El estudiante compara la solución con un caso similar.

\section{Explicación}

La tutora realiza una explicación y para ello ofrece un ejemplo. En este caso, ofrece un ejemplo de un conector ordenador del discurso y no causal, como el que aparece en la oración leída. Es, por tanto, un ejemplo genérico y no específico.

E: Vale. El estudiante muestra la comprensión de la explicación.

T: ¿Vale? "... entre la ida y la vuelta no daría tiempo a hacer ninguna actividad..."
Lectura en voz alta de la tutora.

La tutora continua con la lectura en voz alta del texto.

Fuente: elaboración propia. 


\section{Conclusiones}

Enseñar a escribir en la universidad es todo un reto para quienes se ocupan de ello. No solo porque en ocasiones encuentren la reticencia por parte de la propia institución educativa al no favorecer iniciativas de alfabetización académica y no valorar la incidencia que tiene la expresión escrita en el rendimiento y éxito académico. También, fundamentalmente, por dos motivos más. En primer lugar, porque -si bien son muchas las experiencias exitosas- poner en marcha un proyecto de mejora de la expresión escrita de los estudiantes y de su conciencia como escritores no solo consiste en adoptar una iniciativa concreta o un modelo de enseñanza de la escritura; sino que hay siempre que adaptarlo al contexto académico en donde la mejora se pretende llevar a cabo y esto siempre es sumamente complicado. En segundo lugar, solventado el anterior inconveniente, no es suficiente contar con los mejores medios. Es necesario formar a quienes se responsabilizan de esta tarea y esto implica, ineludiblemente, tiempo.

En el caso de las tutorías de escritura, los tutores son estudiantes de los últimos cursos del grado o de posgrado motivados y comprometidos con el apoyo a sus compañeros, pero que estarán en general poco tiempo vinculados al centro o programa que promueve las tutorías. Cabe puntualizar, al respecto, que no es lo mismo el vínculo que puede mantener un tutor que en el último curso de la carrera ofrece sus primeras tutorías y que, posteriormente, deja de tener relación con la universidad, que otro tutor de posgrado que realiza una maestría o doctorado, y cuya relación con la institución se espera que se prolongue en el tiempo. Por eso, dentro de un continuo que incluye su captación, selección y formación, parte del éxito radica en ofrecer la mejor formación posible a los tutores.

Cómo hacerlo es lo que ha motivado este trabajo, ya que atender a las estrategias didácticas que ponen en práctica los tutores noveles servirá para que tomen conciencia del modelo de tutoría que promueven y, por tanto, de los aspectos positivos en los que han de incidir y de los negativos que deben mejorar. En concreto, los datos del análisis de contenido muestran que la tutora novel tiende a utilizar más estrategias cognitivas que instructivas y motivacionales. No obstante, al igual que en las investigaciones similares precedentes (Cromley y Azevedo, 2005; Mackiewicz y Thompson, 2014, 2018), en sus primeras tutorías destaca un modelo centrado en el tutor y en el producto, y no en el estudiante y el proceso. Así, los estudios citados y los resultados de esta investigación coinciden en aspectos significativos como cierta homogeneidad en el uso de estrategias cognitivas e instructivas en los tutores noveles. También concuerdan en una tendencia hacia la lectura en voz alta (Mackiewicz y Thompson, 2018), en este caso, de la tutora, propia de los tutores con menos experiencia. Cabría preguntarse al respecto si existe la posibilidad de que la tutora novel no haya hecho más que replicar un modelo de tutoría similar al tipo de retroalimentación que ha recibido durante sus estudios universitarios por parte de sus profesores, pese a su formación como docente y también a la recibida en el centro de escritura.

Por otro lado, de acuerdo con Pigliacelli (2017), los resultados sugieren que la formación individualizada basada en la reflexión sobre las propias tutorías podría ser un factor que influyera en la mejora de la calidad de las tutorías. Por eso, la observación a tutores expertos y el análisis de lo que hacen los tutores en sus primeras tutorías a partir de vídeos o audios debe ser un eje en torno al cual gire su formación; además de las reuniones y seminarios que persiguen familiarizar al tutor novel con el centro o programa de escritura, y con la enseñanza del proceso de escritura y los géneros académicos. También, se podría considerar que esta dinámica de análisis puede ser válida para la formación del docente universitario y de los estudiantes a los que se involucran en actividades de revisión entre pares. Por último, dado el crecimiento que tanto en España como en Latinoamérica están teniendo los centros de escritura, la 
atención y el conocimiento de las variables que pueden influir en una tutoría de escritura exitosa constituyen un campo de investigación incipiente en los estudios sobre alfabetización académica en el ámbito hispano.

\section{Reconocimientos}

Este trabajo se ha realizado con la colaboración de Andrea Barrado Mendo, en el marco de la Beca de Colaboración en Departamentos Universitarios para el curso académico 2019-2020 del Ministerio de Educación y Formación Profesional. También ha contado con la colaboración de Adrián Neubauer Esteban, estudiante del Doctorado de Educación con contrato predoctoral de Formación de Personal Investigador de la Universidad Autónoma de Madrid.

\section{Referencias bibliográficas}

Álvarez, G. y Difabio de Anglat, H. (2018). Retroalimentación docente y aprendizaje en talleres virtuales de escritura de tesis. Apertura, 10(1), 8-23. DOI: http://dx.doi.org/10.32870/Ap.v10n1.996

Álvarez, I., Espasa, A. y Guasch, T. (2011). The value of feedback in improving collaborative writing as- signments in an online learning environment. Studies in Higher Education, 37(4), 387-400. DOI: 10.1080/03075079.2010.510182

Alzate, G. M. y Peña, L. B. (2010). La tutoría entre iguales: una modalidad para el desarrollo de la escritura en la educación superior. Universitas Psychologica, 9(1), 123-138.

Bach, C. y Montané, A. (2016). Mentories de Ilengua per a la millora de les competències de redacció acadèmica. Revista CIDUI, 3, 1-10. Recuperado de https://www.cidui.org/revistacidui/index.php/cidui/ article/view/975/941

Boyd, J. L. (2013). Writing centers and the problem of expertise: knowing and doing in peer tutoring. [Tesis doctoral]. Indiana University of Pennsylvania, Indiana.
Caldwell, E., Stapleford, K. y Tinker, A. (2018). Talking academic writing: a conversation analysis of oneto-one learning development tutorials. Journal of Academic Writing, 8(2), 124-136. DOI: 10.18552/ joaw.v8i2.464

Castellà, J. M., y Aliagas, C. (2016). La mentoría entre iguales para el aprendizaje de la escritura académica. Una experiencia en la Universidad Pompeu Fabra de Barcelona. En G. Bañales, M. Castelló y N. A. Vega (eds.), Enseñar a leer y a escribir en la educación superior. Propuestas educativas basadas en la investigación. (pp. 281-314). Ciudad de México: SM.

Chois, P. M., Casas, A. C., López, A., Prado, D. M. y Cajas, E. Y. (2017). Percepciones sobre la tutoría entre pares en escritura académica. Magis. Revista Internacional de Investigación en Educación, 9(19), 165-184. DOI: https://doi.org/10.11144/Javeriana. m9-19.ptpe

Corcelles, M., Cano, M., Bañales, G. y Vega, N. A. (2013). Enseñar a escribir textos científico-académicos mediante la revisión colaborativa: el trabajo final de grado en Psicología. Revista de Docencia Universitaria, 11(1), 79-104. DOI: 10.4995/ redu.2013.5593

Cromley, J. G. y Azevedo, R. (2005). What do reading tutors do? A naturalistic study of more and less experienced tutors in reading. Discourse Processes, 40(2), 83-113. DOI: 10.1207/s15326950dp4002_1

Dinitz, S. y Kiedaisch, J. (2003). Creating theory: moving tutors to the center. The Writing Center Journal, 23(2), 63-76.

Durán, D. (2006). Tutoría entre iguales, la diversidad en positivo. Aula de Innovación Educativa, 153, 7-10.

Eastmond, L. (2019). Scaffolding in the center: training tutors to facilitate learning interactions with L2 writers. [Tesis doctoral]. Arizona State University, Phoenix.

Errázuriz, M. C. (2017). Las tutorías de un centro de escritura como dispositivo de modelamiento de estudiantes de programas de formación inicial docente. En L. Natale y D. Stagnaro (eds.), Alfabetización académica: un camino para la inclusión en el nivel superior (pp. 103-131). Los Polvorines: UNGS. 
Fitzgerald, L. y lanetta, M. (2016). The Oxford guide for writing tutors: practice and research. Oxford: Oxford University Press.

García-Arroyo, M. y Quintana, H. (2016). La capacitación profesional de los tutores en los centros de escritura. En G. Bañales, M. Castelló y N. A. Vega (eds.), Enseñar a leer y a escribir en la educación superior. Propuestas educativas basadas en la investigación. (pp. 364-389). Ciudad de México: SM.

Gilewicz, M. y Thonus, T. (2003). Close vertical transcription in writing center training and research. The Writing Center Journal, 24(1), 25-49.

Harrington, K., O'Neill, P. y Bakhshi, S. (2007). Writing mentors and the Writing Centre: producing integrated disciplinary writers. Investigations in University Teaching and Learning, 4(2), 26-32.

Kirchhoff, L. (2016). Motivation in the Writing Centre: A Peer Tutor's Experience. Journal of Academic Writing, 6(1), 31-40. DOI: 10.18552/joaw.v6i1.282

Mackiewicz, J. (2004). The effects of tutor expertise in engineering writing: a linguistic analysis of writing tutors' comments. IEEE Transactions on Professional Communication, 47(4), 316-328. DOI: 10.1109/ TPC.2004.840485

Mackiewicz, J. (2005). Hinting at what they mean: indirect suggestions in writing tutors' interactions with engineering students. IEEE Transactions on Professional Communication, 48(4), 365-376. DOI 10.1109/TPC.2005.859727

Mackiewicz, J. y Thompson, I. (2014). Instruction, cognitive scaffolding, and motivational scaffolding in writing center tutoring. Composition Studies, 42(1), 54-78.

Mackiewicz, J. y Thompson, I. (2018). Talk about writing. The tutoring strategies of experienced writing center tutors. Nueva York/Londres: Routledge.

Molina-Natera, V. (2019). El discurso pedagógico en las tutorías de escritura. Develando elementos de una práctica educativa. Revista Mexicana de Investigación Educativa, 24(80), 125-148.

Monty, R. W. (2013). Theoretical communities of praxis: the university writing center as cultural contact zone. [Tesis doctoral]. The University of Texas at El Paso, El Paso.
Murphy, C. y Sherwood, S. (2008). The St. Martin's sourcebook for writing tutors. Londres: Macmillan Education.

Natale, L. (2014). Interrelaciones entre representaciones discursivas sobre la escritura académica y devoluciones escritas de docentes universitarios. Onomázein, (número extra 1), 81-98.

Natale, L. (2016). Las devoluciones escritas del profesor universitario. En L. Natale y D. Stagnaro (orgs.), La lectura y la escritura en las disciplinas. Lineamientos para su enseñanza (pp. 167-200). Los Polvorines: UNGS.

North, S. M. (1982). Training Tutors to Talk about Writing. College Composition and Communication 33(4), 434-441. DOI: 10.2307/357958

Núñez, J. A. y Errázuriz, M. C. (2020). Panoramas de la alfabetización académica en el ámbito iberoamericano: aportes para la calidad de la Educación Superior. Tendencias Pedagógicas, 36, 1-8. DOI: https://doi.org/10.15366/tp2020.36.01

Piaget, J. (1970). The science of education and the psychology of the child. Nueva York: Orion Press.

Pigliacelli, M. (2017). Practitioner action research on writing center tutor training: critical discourse anal$y$ sis of reflections on video-recorded sessions. [Tesis de maestría]. Nueva York: Long Island University.

Roland, K. A. (2008). Educating for inclusion: community buelidng through mentorship and citizenship. The Journal of Educational Thought, 42(1), 53-67.

Roldán, C. A. y Arenas, K. A. (2016). Características de las tutorías del Centro de Lectura y Escritura de la Universidad Autónoma de Occidente: ¿Qué muestran los registros de atención? Revista Grafía,13(1), 100-114. DOI: http://dx.doi. org/10.26564/16926250.658

Ryan, L. y Zimmerelli, L. (2006). The Bedford Guide for Writing Tutors. Boston: Bedford/St. Martin's Press.

Scardamalia, M. y Bereiter, C. (1992). Dos modelos explicativos de los procesos de composición escrita. Infancia y Aprendizaje, 15(58), 43-64.

Thompson, I. (2009). Scaffolding in the writing center: a microanalysis of an experienced tutor's verbal and nonverbal tutoring strategies. Written Communication, 26(4), 417-453. DOI: 10.1177/0741088309342364 
Thonus, T. (2002). Tutor and student assessments of academic writing tutorials: What is "success"? Assessing Writing, 8(2), 110-134. DOI: 10.1016/ S1075-2935(03)00002-3

Uribe, G., Mateo, M. T., Agosto, S. E. y Álvarez, T. (2020). El miniensayo y su didáctica: escribir en las materias del currículo. Barcelona: Octaedro.

Vygotsky, L. (1978). Mind in society. The development of higher psychological processes. Cambridge: Harvard University Press.
Weaver, M. (2006). Do students value feedback? Student perceptions of tutors' written responses. Assessment \& Evaluation in Higher Education, 31(3), 379-394. DOI: 10.1080/02602930500353061

Wingate, M. (2005). What line? I didn't see any line. En B. Rafoth (ed.), A tutor's guide: helping writer's one to one (pp. 9-16). Portsmouth: Boynton. 